\title{
ESTUDIO DEL CONSUMO Y APORTE NUTRICIONAL DE BOCADILLOS EN ESCOLARES DE LA REGIÓN METROPOLITANA DE CHILE
}

\section{STUDY OF SNACKS CONSUMPTION AND THEIR NUTRITIONAL COMPOSITION LEVELS OF SCHOOL AGE CHILDREN OF METROPOLITAN REGION OF CHILE}

\author{
Marcela Zamorano R., Ernesto Guzmán C., Jorge Ibáñez \\ Departamento de Ciencia y Tecnología de los Alimentos, \\ Facultad Tecnológica, Universidad de Santiago de Chile, USACH. Santiago. Chile.
}

\begin{abstract}
Snack foods are products easy to handle. The objective of this work was to determine their consumption, proximate composition and fatty acid profile of snack food consumed by schoolage children of Metropolitan Region of Chile. To reach these goals, 203 school-children were interviewed to determine snack foods consumption frequency and preference. Results showed that, this type of food represents an important pattern in diet. The highest snack consumption were potatoes chips type american and plain, chocolate cookies (small package) and corn chips. Proximate composition and fatty acids profile were determinate for these foods, each analysis was carried out in duplicate and three samplings were performed. Four snack foods analyzed had a significant fat content near 25 $\mathrm{g} / 100 \mathrm{~g}$ and low contents of protein and total dietary fiber, indicating that these foods are products with high fat and caloric intake. For fatty acids profile, cookies presented the highest content of SFA, with 63 g/100 for PUFA content, the potato chips plain showed a high content, $60,3 \mathrm{~g} / 100 \mathrm{~g}$. These products present a high contribution in fat and energy, their high frequency consumption could be an important factor in the high rates of obesity observed in recent years in schoolage-children in the Metropolitan Region of Chile.

Key words: snack food, scholl population, proximate analysis, caloric intake, fatty acid profile.
\end{abstract}

Este trabajo fue recibido el 25 de Noviembre de 2009, aceptado con modificaciones el 8 de Junio de 2010 y aceptado para ser publicado el 20 de Octubre de 2010.

\section{INTRODUCCIÓN}

Durante los últimos años la población Chilena ha experimentado notables cambios en sus hábitos alimentarios, mostrando un aumento creciente en el consumo de alimentos industrializados debido principalmente al acelerado ritmo de vida que existe actualmente, lo que lleva a las personas a destinar cada vez menos tiempo en la preparación de sus comidas. Es así, como la oferta de alimentos llamados "Snack" o bocadillos, que se definen como productos fáciles de manipular, constituidos en porciones individuales, que no requieren preparación y satisfacen el apetito en corto plazo y los cuales no debieran superar el $10 \%$ del total de calorías requeridas en un día, se ha impuesto en la dieta alimentaria habitual de los chilenos.

En este sentido, la población escolar parece tener un alto consumo de este tipo de alimentos, lo que se refleja en su alta venta en los colegios. Investigaciones que han incursionado en el gasto que realizan los escolares y el dinero que invierten en comprar sus alimentos han demostrado que el $68.1 \%$ de ellos compran productos envasados dulces o salados, tipo bocadillo, un $17,2 \%$ bebidas gaseosas y sólo el 6,3\% yogur o leche (1). Hoy en día, este grupo etáreo muestra una tendencia creciente a la prevalencia de sobrepeso y obesidad, lo que es corroborado por investigaciones que han coincidido que existe una ingesta insuficiente de lácteos, verduras y frutas y un elevado consumo de alimentos de alta den- 
sidad calórica, siendo esto un factor determinante para las altas tasas de sobrepeso y obesidad en escolares. (2).

En Chile existe poca información sobre la composición química y nutricional que tienen este tipo de alimentos, salvo la entregada por las propias empresas. La única información disponible esta basada en la Tabla de Composición Química de los Alimentos Chilenos, herramienta que ha sido utilizada por numerosos profesionales del área de alimentos y nutrición pero que sin embargo no ha sido actualizada y casi no tiene información sobre alimentos tipo bocadillo. En los últimos años la existencia de la Red Internacional de Datos sobre Alimentación (INFOODS) y la Red Latinoamericana de Composición de Alimentos (LATINFOODS), ambos sistema de datos de alimentos dependiente de la Organización de las Naciones Unidas para la Agricultura y la Alimentación (FAO) y disponible en Internet, ha incorporado información sobre alimentos y componentes, no obstante en lo que se refiere a este tipo de alimentos solo se mencionan algunas galletas, información también reportada por Gattás el año 2008, en la que entrega datos sólo sobre galletas de una marca específica y hojuelas de papas fritas, información no evaluada y recolectada de empresas y la tabla de composición química chilena y española. (4).

En base a estos antecedentes esta investigación plantea como objetivo determinar la incidencia del consumo, composición química, aporte calórico y perfil de ácidos grasos de alimentos tipo bocadillo de mayor consumo en la población escolar de la Región Metropolitana.

\section{SUJETOS Y MÉTODOS}

Encuesta: Se realizó una encuesta alimentaria a 290 escolares pertenecientes a colegios de 13 comunas de la Región Metropolitana de diferente nivel socioeconómico y académico, para lo cual se realizó una elección aleatoria de establecimientos considerando la ecuación propuesta por Scheaffer( 1987), en el que se encuestó a un grupo elegido al azar de un curso, que varió desde primero básico a octavo básico (12). Los escolares fueron entrevistados, por personal entrenado y supervisión del profesor jefe, con un cuestionario estructurado que contenía 7 preguntas sobre la frecuencia y preferencia de consumo de alimentos tipo bocadillo, para lo que se propusieron 3 alternativas en relación al consumo, además permitió conocer el tipo y marca de productos que prefieren los escolares. Para esto se eligió un universo de 8 productos definidos como "bocadillo", como son papas fritas "chips", ramitas, chips de maíz, suflés, chips de queso, cabritas, galletas y barras de cereales, adicionalmente se agregaron subgrupos para tener un detalle de las preferencias debido a la gran variedad que tiene cada grupo.

\section{Materiales}

El procesamiento y análisis de las encuestas permitió la selección de los productos y marcas de mayor consumo de la población escolar de la Región Metropolitana. De esta forma se seleccionaron a 4 tipos de bocadillos para este estudio, dos marcas de papas fritas chips, una corte americano y otra corte liso, 1 marca de galletas horneadas con chispas de chocolate y 1 marca de chips de maíz. Se procedió a un muestreo aleatorio y para cada producto seleccionado se obtuvieron muestras recolectadas de distintas cadenas de supermercados, minimarket y quioscos, de diferentes zonas de la Región Metropolitana. Para la determinación del N muestral se consideró a Holden (1997) que indica la relación del tamaño de la muestra $(\mathrm{N})$ con el coeficiente de variación y error estándar de las metódicas analíticas (11). Para la obtención de las unidades se realizó un muestreo mensual, en que cada una de las muestras estuvo constituida por 5 unidades que generaron una muestra analítica. Este se realizó por tres meses en temporada primavera verano, controlándose el número de lote para asegurarse de que cada unidad perteneciera a distintos lotes, el total de muestras recolectadas fue de 15 por producto.

Las muestras obtenidas fueron trasladadas al lugar de análisis en sus envases originales. En el lugar de análisis se registro el nombre, origen, fecha y peso. Estas fueron homogenizadas al llegar al laboratorio, para ello se utilizó un procesador de alimento (Moulinex, modelo D56), luego se dividió en dos porciones que se guardaron en bolsas herméticas. Una de las muestras guardadas se conservó como contramuestra y la otra se subdividió en dos partes, una de estas se usó para realizar el análisis proximal y la otra para la determinación de materia grasa y perfil de ácidos grasos.

\section{Métodos}

La determinación de humedad y cenizas se realizó mediante desecación, incineración y gravimetría según norma AOAC, Part 925.09 y 923.03 (4). Proteínas por el método de Kjeldahl para determinación de nitrógeno aplicando factor 6,25 según AOAC, Part 991.20. La grasa total se extrajo utilizando el método de WernerSchmidt con posterior determinación gravimétrica del sedimento (6). La fibra dietaria total fue determinada utilizando el método enzimático gravimétrico, AOAC, Part 991.43. Los carbohidratos disponibles fueron determinados por diferencia según la recomendación de INFOODS (7). El aporte calórico fue obtenido aplicando factores de Atwater de $4 \mathrm{kcal} / \mathrm{gr}$ para proteínas, $9 \mathrm{kcal} / \mathrm{gr}$ para grasa y $4 \mathrm{kcal} / \mathrm{gr}$ para hidratos de carbono.

Para la determinación del perfil de ácidos grasos, se extrajo la grasa mediante el método de Bligh and 
Dyer según AOAC; Part. 983.23, para luego obtener los ácidos grasos, previa metilación con trifluoruro de boro en metanol (AOAC, Part 969.63.), los que fueron separados y cuantificados utilizando un cromatógrafo Perkin Elmer, modelo Autosystem, equipado con un detector de ionización de llama, con una columna SP2330 de $30 \mathrm{mt}$ x $0,25 \mathrm{~mm}$ id. (Supelco).

Los resultados se expresaron como el promedio de las determinaciones analíticas de los tres subgrupos constituidos para cada producto. Todos los análisis se realizaron en duplicado.

\section{RESULTADOS Y DISCUSIÓN}

La encuesta realizada demostró que del total de escolares encuestados, el 99\% consume alguna clase de bocadillo durante la semana. En cuanto al tipo de bocadillo que presenta mayor predilección la figura 1 indica que las papas fritas tipo "chips" presentan la primera opción de las preferencias $(28,1 \%)$, luego con un $24,1 \%$ las galletas en paquete individual, seguidos por productos como chips de maíz, barras de cereales, ramitas y suflés y otros productos con porcentajes menores de preferencia. Además a los encuestados, se les preguntó sobre las marcas comerciales y tipos particulares de productos que concitaban su mayor preferencia entre los bocadillos lo que permitió seleccionar los tipos de bocadillo que se analizaron al individualizar marcas específicas. Estos resultados, manifestaron una muy alta preferencia para papas fritas "chips" tipo corte americano, luego en una preferencia mas baja para el tipo de corte liso y en el caso de las galletas, la mayor predilección la tuvo la galleta horneada con chips de chocolate. Además se observó que los chips de maíz, un producto relativamente nuevo en el mercado, presentan una preferencia de un $14,8 \%$. En todos estos casos se indicó además una marca comercial determinada para cada uno de ellos. De acuerdo con estos resultados, se seleccionaron a dichos cuatro alimentos tipo bocadillo para ser analizados.

\section{Composición proximal de los diferentes "bocadillos" estudiados}

En la tabla 1 se muestra la composición química proximal de los cuatro tipos de bocadillos analizados. Todas las muestras estudiadas presentan un alto contenido de grasa entre un 22 a 31 g/100g, observándose los valores más altos en los dos tipos de chips de papas fritas estudiados, valor esperable debido al procesamiento por fritura aplicado en su producción. Las galletas horneadas y los chips de maíz presentan contenidos de grasa cercanos a $23 \mathrm{~g} / 100 \mathrm{~g}$, constituyéndose también en alimentos con un aporte importante de grasa lo que conlleva a elevar también su aporte energético . Los contenidos de humedad, cenizas, proteínas y fibra dietética total, de los 4 alimentos analizados, están dentro de los rangos habituales para ellos, reportados en Gattás(2008) $y$ en LATINFOODS (7), mostrando un aporte proteico relativamente bajo entre 5,6 a $7 \mathrm{~g} / 100 \mathrm{~g}$, mostrando el

\section{FIGURA 1}

Preferencia (\%) de alimentos tipo bocadillo mas consumidos por los escolares de la Región Metropolitana.

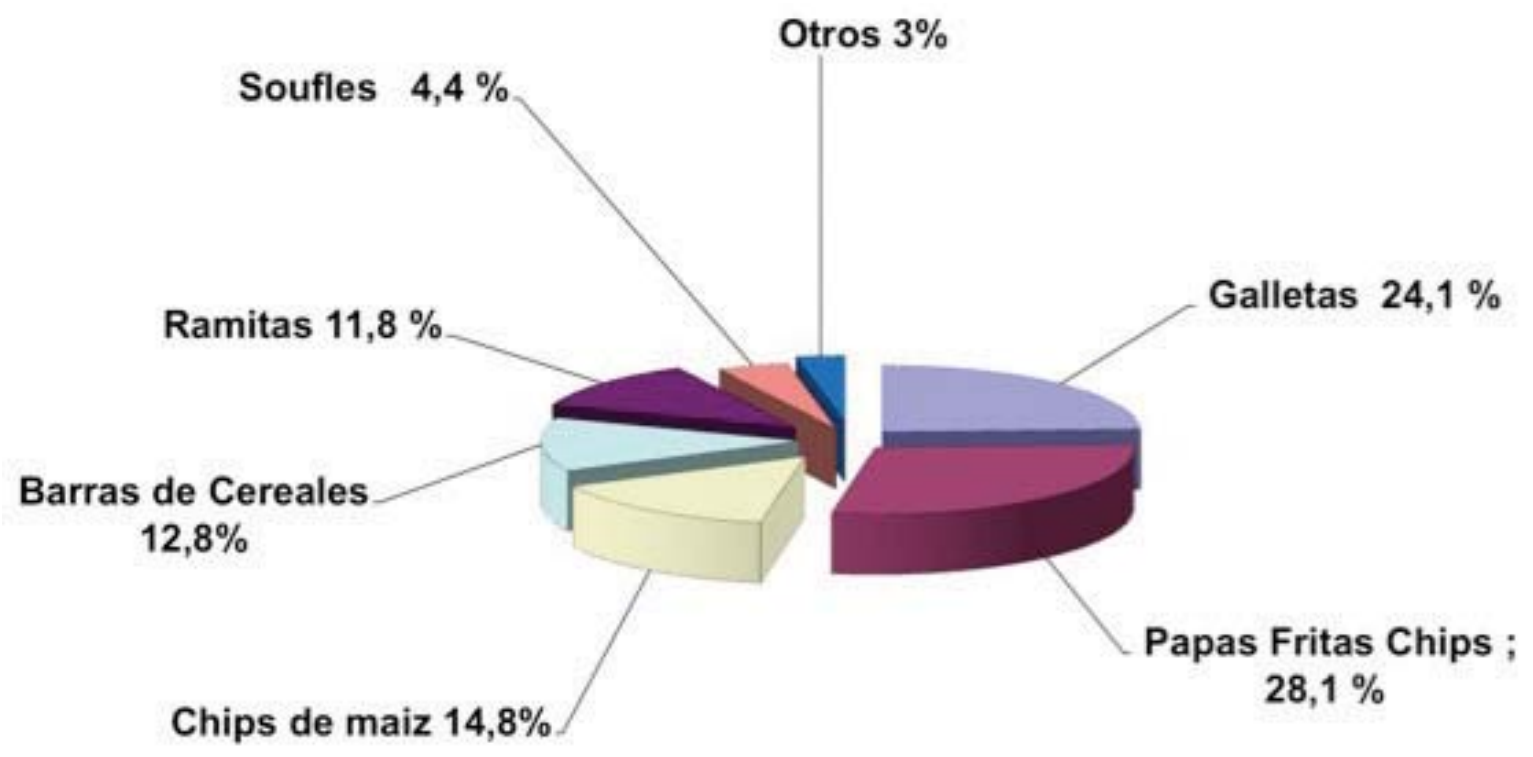


valor mas alto los chips de maíz. El contenido de cenizas se reporta algo elevado en los bozadillos salados $(2,3$ $-3,9 \mathrm{~g} / 100 \mathrm{~g}$ ), esperable por la presencia de cloruro de sodio en ellos. El contenido de fibra dietaria total, fluctuó entre 2,1 a 4,5g/100g, no existiendo referencias para su comparación, sin embargo es un valor esperado por el origen vegetal de estos bocadillos. El aporte calórico obtenido para estos cuatro alimentos tipo bacadillo tiene un rango de 481 a $512 \mathrm{Kcal}$ por $100 \mathrm{~g}$, lo que ratifica ser clasificados como alimentos de alta densidad calórica., en especial si son consumidos como parte de colación por escolares.

La figura 2 muestra los aportes de grasa y energía por porción de consumo, observándose similitud entre los

\section{TABLA 1}

Composición química proximal (por 100 g) de cuatro alimentos tipo bocadillo.

\begin{tabular}{lcccc} 
Nutriente & $\begin{array}{c}\text { Papas fritas } \\
\text { chips corte } \\
\text { americano }\end{array}$ & $\begin{array}{c}\text { Papas fritas } \\
\text { chips } \\
\text { corte liso }\end{array}$ & $\begin{array}{c}\text { Galletas } \\
\text { horneadas con } \\
\text { chispas de chocolate }\end{array}$ & $\begin{array}{c}\text { Chips } \\
\text { de } \\
\text { maíz }\end{array}$ \\
\hline Humedad $(\mathrm{g} / 100 \mathrm{~g})$ & $2,3 \pm 0,2 \mathrm{a}$ & $2,4 \pm 0,1 \mathrm{a}$ & $2,0 \pm 0,1 \mathrm{a}$ & $1,7 \pm 0,08 \mathrm{a}$ \\
Ceniza $(\mathrm{g} / 100 \mathrm{~g})$ & $3,9 \pm 0, \mathrm{a}$ & $3,4 \pm 0,3 \mathrm{a}$ & $1,2 \pm 0,1 \mathrm{a}$ & $2,3 \pm 0,2 \mathrm{a}$ \\
Proteína $(\mathrm{g} / 100 \mathrm{~g}) \mathrm{b}$ & $5,6 \pm 0,3 \mathrm{a}$ & $6,4 \pm 0,3 \mathrm{a}$ & $6,2 \pm 0,3 \mathrm{a}$ & $7,0 \pm 0,1 \mathrm{a}$ \\
Grasa $(\mathrm{g} / 100 \mathrm{~g})$ & $31,1 \pm 1,2 \mathrm{a}$ & $29,7 \pm 1,7 \mathrm{a}$ & $23,9 \pm 1,0 \mathrm{a}$ & $22,7 \pm 1,2 \mathrm{a}$ \\
Fibra dietaria total $(\mathrm{g} / 100 \mathrm{~g})$ & $4,5 \pm 0,2 \mathrm{a}$ & $4,0 \pm 0,08 \mathrm{~b}$ & $2,1 \pm 0,2 \mathrm{c}$ & $3,9 \pm 0,3 \mathrm{a}$ \\
Hidratos de carbono disponibles((g/100g) & $52,6 \pm 1,7 \mathrm{a}$ & $54,1 \pm 2,0 \mathrm{~b}$ & $64,6 \pm 1,4 \mathrm{a}$ & $62,4 \pm 1,0 \mathrm{a}$ \\
Energía (kcal/100g) & 512,7 & 509,3 & 497,9 & 481,9
\end{tabular}

a Cada valor es el promedio de $\mathrm{n} \pm$ D.S, $\mathrm{n}=3$ muestra compuesta $=5$, análisis en duplicado.

b Nitrógeno Kjeldahl x6.25. Letras diferentes en la columna son diferencias significativas entre los muestreos con p $<0,05$ por Anova.

\section{FIGURA 2}

Aporte de grasa (g) y energía (Kcal) en porción de consumo de los cuatro tipos de bocadillos analizados.

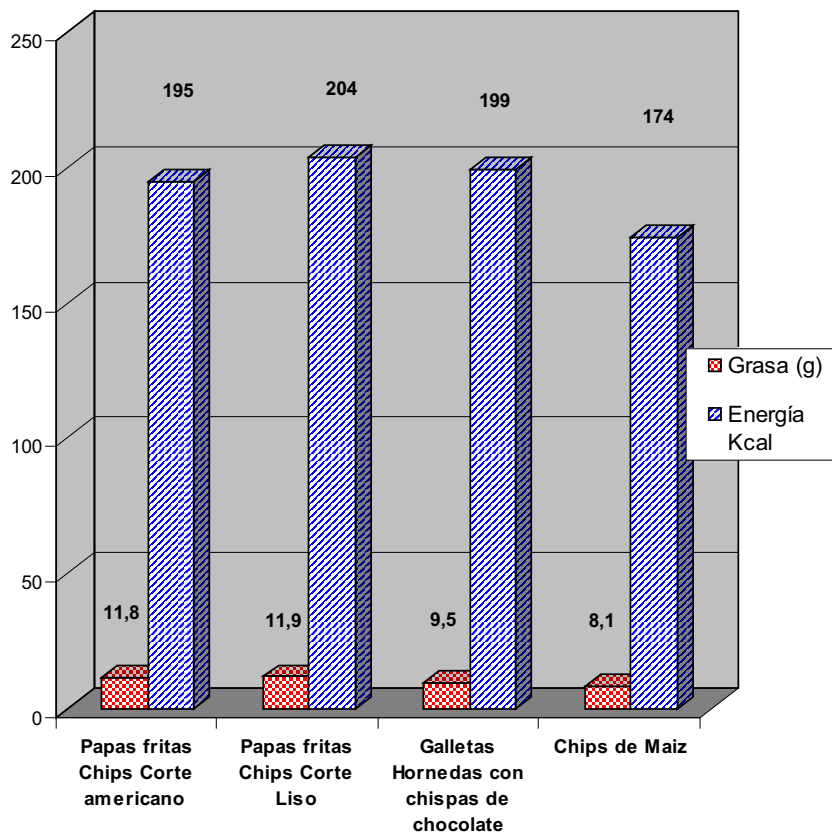


cuatro tipos de bocadillos analizados, con aportes calóricos por porción del orden de $190 \mathrm{Kcal}$, lo que significa alrededor del $10 \%$ de la energía diaria requerida para un niño entre 9 y 10 años con actividad física moderada. Sin embargo en niños mas pequeños o con actividad física liviana, un aporte de 190 calorías entregados por estos bocadillos que se consumen en forma adicional a las cuatro comidas diarias y con una frecuencia de consumo de al menos tres veces a la semana, podrían tener un rol importante en la obesidad y sobrepeso de escolares de la Región Metropolitana de Chile.

\section{Perfil de ácidos grasos de los diferentes "bocadillos" estudiados}

La tabla 2 muestra el perfil de ácidos grasos de los 4 alimentos tipo bocadillo estudiados, mostrando diferencias significativas $(p \leq 0.05)$ entre ellos. Se observa que para los chips de papas fritas, las de corte americano presentaron un contenido de ácido oleico (C18:1) de 66,4 $\%$ y de $24,8 \%$ los chips de corte liso. En cuanto al contenido de ácidos grasos saturados (AGS), ambos tipos de chips de papas fritas presentan contenidos similares del orden del $15 \%$. Sin embargo, la mayor diferencia se muestra en el contenido de ácidos grasos poliinsaturados, (AGPI), en donde los chips de corte americano presenta un contenido muy bajo, de un $17,9 \%$, en comparación con los chips corte liso que tiene un $60,3 \%$ de este tipo de ácidos grasos. Esto es claramente indicativo del tipo de grasa utilizado en el proceso de fritura, que para los chips corte liso correspondería a aceites vegetales. Esto produce un aumento notable de la razón entre ácidos grasos poliinsaturados y ácidos grasos saturados (AGPI/

\section{TABLA 2}

Perfil en ácidos grasos de cuatro alimentos tipo bocadillo (expresado en \% de esteres metílicos).

\section{$\%$ de esteres metílicos}

Ácidos grasos saturados (AGS)

Ac. caprílico C 8:0

Ac. capricho C10:0

Ac láurico $\mathrm{C} 12: 0$

Ac mirístico C14:0

Ac palmítico C16:0

Ac esteárico C18:0

Ac. eicosanoico C20:0

Total

Ácidos grasos

saturados(AGM)

Ac oleico C18:1

Ac erúcico C 22:1

Total

Ácidos grasos

polinsaturados(AGPI)

Ac linoleico C18:2

Ac linolénico C 18:3

Total

Relación (AGPI/AGS)

\section{Papas fritas chips corte \\ americano}

Papas fritas
chips
corte liso

corte liso

Galletas
horneadas con
chispas de chocolate

\section{Chips}

de

maíz

$\begin{array}{cccc}\text { nd } & \text { nd } & 2,6 \pm 1,0 & \text { nd } \\ \text { nd } & \text { nd } & 2,0 \pm 0,7 & 0,1 \pm 0 \\ 0,2 \pm 0,08 & \text { nd } & 17,5 \pm 5,7 & 0,2 \pm 0,05 \\ 0,2 \pm 0,04 & 0,1 \pm 0 & 5,7 \pm 0,9 & 0,4 \pm 0,07 \\ 10,4 \pm 0,9 & 11,0 \pm 0,5 & 31,4 \pm 2,1 & 10,6 \pm 0,5 \\ 4,3 \pm 1,0 & 3,2 \pm 0,4 & 4,4 \pm 0,5 & 4,3 \pm 1,1 \\ 0,5 \pm 0,1 & 0,4 \pm 0,1 & 0,1 \pm 0,05 & 0,5 \pm 0,08 \\ \mathbf{1 5 , 6} & \mathbf{1 4 , 7} & \mathbf{6 3 , 7} & \mathbf{1 6 , 1}\end{array}$

a Cada valor es el promedio de $\mathrm{n} \pm$ D.S, $\mathrm{n}=3$ muestra compuesta $=5$, análisis en duplicado nd $=$ no detectado. 
AGS) la que según $\mathrm{FAO}(8)$, debiera tener un valor sobre 4 para una materia grasa de buena calidad. Los chips de maíz, presentaron un perfil de ácidos grasos comparable al de los chips de papas fritas corte americano, con altos contenidos de ácidos grasos monoinsaturados de 62,7\% y aporte del $21,2 \%$ para ácidos grasos poliinsaturados, lo que es esperado por originarse ambos de la misma industria.

Las galletas horneadas presentan un alto contenido de ácidos grasos saturados, del orden del $63,7 \%$ y por lo tanto bajos aportes, de 25,6\% para ácidos grasos monoinsaturados y de 10,7 \% de poliinsaturados. En este contexto, este bocadillo sería el que posee una materia grasa de más mala calidad, con una razón de AGPI/AGS menor a 1. Este perfil denota la utilización en la fabricación de estas galletas de grasas altamente saturadas como son manteca de cacao, grasa láctea y coco. Considerando, entonces las porciones de consumo de estos bocadillo y el aporte de grasa de ellos, se deduce que el bocadillo que presenta mejor calidad de la grasa sería los chips de papas fritas corte liso, aportando 7,17 g de ácidos grasos polinsaturados por porción de consumo habitual. Sin embargo este valor cubriría casi el $50 \%$ de la recomendación de consumo de AGPI para un niño entre 6 a10 años, por lo que sería recomendable disminuir su frecuencia de consumo (8). Esta situación podría ser mas desfavorable, al considerar que estos alimentos procesados podrían incluir grasas trans (AGT), las que se asocian a dislipidemias. Se pretende en investigaciones posteriores incluir a este tipo de ácidos grasos, no sólo en alimentos tipo bocadillo, si no realizar una revisión mas amplia de muchos alimentos consumidos por la población chilena.

\section{CONCLUSIONES}

Los alimentos denominados bocadillos son productos de alto consumo por escolares de distintos niveles educacionales y socioeconómicos de la Región Metropolitana de Chile. Su frecuencia de consumo es más de tres veces por semana, y los de mayor demanda son los chips de papas fritas. Los cuatro alimentos tipo de bocadillo estudiados presentan un alto contenido de materia grasa con valores cercanos al $25 \mathrm{~g} / 100 \mathrm{~g}$, además de tener bajos contenidos de proteínas y fibra dietaria total, considerando a este tipo de alimento como un producto con alto contenido graso y aporte calórico. El perfil de ácidos grasos demostró, en tres de los cuatro tipos de bocadillos analizados, un alto contenido de AGS, mostrando las galletas un valor de AGS cercano a $64 \mathrm{~g} / 100 \mathrm{~g}$ de grasa. Solo los chips de papas fritas corte liso mostraron una adecuada relación AGPI/AGS.

\section{RESUMEN}

Los alimentos tipo bocadillo son productos fáciles de manipular que no requieren preparación. El objetivo de esta investigación fue determinar la incidencia del consumo, composición química, aporte calórico y perfil de ácidos grasos de alimentos tipo bocadillo de mayor consumo en la población escolar de la Región Metropolitana de Chile. Se realizó una encuesta a 203 escolares para determinar su frecuencia y preferencia. Los resultados indicaron un alto consumo, mostrando mayor preferencia por cuatro tipos de bocadillo, chips de papas fritas corte americano y liso, galletas con chispas de chocolate en paquete pequeño y chips de maíz. A estos alimentos se les determinó análisis proximal y perfil de ácidos grasos, para lo cual se realizó 3 muestreos con muestras compuestas y análisis en duplicado. Los cuatro alimentos tipo de bocadillo estudiados presentan un alto contenido de materia grasa con valores cercanos al $25 \mathrm{~g} / 100 \mathrm{~g}$, además de tener bajos contenidos de proteínas y fibra dietaria total llevando a considerar por consiguiente a este tipo de alimento como productos con un alto contenido graso y aporte calórico. El perfil de ácidos grasos demostró, en tres de los cuatro tipos de bocadillo analizados, un alto contenido de AGS, mostrando la grasa de las galletas un valor de AGS cercano a $64 \mathrm{~g} / 100 \mathrm{~g}$, solo los chips de papas fritas corte liso mostraron una adecuada relación AGPI/AGS. Estos productos presentan un alto aporte en grasa y energía, su alta frecuencia de consumo podría ser un factor importante en los altos índices de obesidad observados en los últimos años en escolares de la Región Metropolitana.

Palabras clave: bocadillo, escolares, análisis proximal, calorías, perfil de ácidos grasos.

Dirigir la correspondencia a:

Profesora

Marcela Zamorano R.

Departamento de Ciencia y Tecnología

de los Alimentos,

Facultad Tecnológica,

Universidad de Santiago de Chile, USACH.

Avda. Ecuador 3769

Estación Central,

Santiago,

Chile

Fono: 7180549

Fax 6823536

E-mail: marcela.zamoran@usach.cl

Agradecimientos: Los autores agradecen el financiamiento de DICYT de la VRID de la USACH. 


\section{BIBLIOGRAFÍA}

1. Olivares S, Yañez R, Diaz N .Publicidad de alimentos y conductas alimentarías en escolares de $5^{\circ}$ a $8^{\circ}$ año Básico. Rev Chil Nutr. 2003; 30 (1):36-42.

2. Olivares $\mathrm{S}$, Bustos $\mathrm{N}$ el al. Estado Nutricional, consumo de alimentos y actividad física en escolares mujeres de diferente nivel socioeconómico de Santiago de Chile. Rev Méd Chil. 2007; 135: 71-8.

3. Burrows R, Diaz E et al. Hábitos de ingesta y actividad física en escolares, según tipo de tipo de establecimiento que asisten. Rev. Méd. Chile. 2008; 136:53-63.

4. Gattas, V. Guía de la Composición Nutricional de Alimentos naturales, de la industria y Preparaciones Chilenas habituales. Universidad de Chile. INTA. 2008.

5. Association of Official Agricultural Chemist. Official Methods of Analysis of AOAC.16th ed. Washington, D.C. The Association, 1995.

6. James, C.S. Analytical Chemistry of Foods. (Ed). Chapman \& Hall. Oxford 1995, p 170.

7. INFOODS (1994). Tagnames for Food Components. Revisado Mayo 21, 2005: http:// fao.org/infoods/
tgas/Otags.htm. LATINFOODS.Tabla de Composición de Alimentos de América Latina, 1990, Revisado en May 12, 2005: http://www.rlc.fao.org/ bases/alimento.ht

8. FAO. Grasas y aceites en la nutrición humana. Estudio FAO Alimentación y Nutrición 57. Roma. 1997.

9. Schmidt - Hebbel H. et al. Tabla de Composición Química de los Alimentos Chilenos. $7^{\text {a }}$ Edición. Ed. Universitaria. Santiago. 1985.

10. Monro J. and Burlingame B. Carbohidratos y Componentes Alimentarios Relacionados: Identificadores de INFOODS, Significados y Usos. En: Producción y manejo de datos de composición química de alimentos en nutrición. (Morón C et al. Ed). FAO., Santiago.1997. p 327-36.

11. Holden M, Joanne, Davis Carol. Estrategias para muestreo: El aseguramiento de valores representativos. En: Producción y manejo de datos de composición química de alimentos en nutrición. (Morón C et al. Ed). FAO., Santiago.1997. p 115-35.

12. Scheaffer R.,Mendenhall W, Ott L. Elementos de muestreo. Ed. Iberoamericana S.A. México D.F.1987. 